\title{
Hanns Eislers Johann Faustus
}

Dieses Kapitel widmet sich einer der ersten Reaktionen auf Thomas Manns Roman, nämlich Hanns Eislers Johann Faustus (1952). Aufgrund des Druckverbots in der DDR vervollständigte Eisler sein Opernprojekt nie: Das Kapitel rekonstruiert knapp die Geschichte des Werkes und geht der Frage nach dem Status des Librettos nach, das in dieser Studie in Anlehnung an Friederike Wißmann für einen selbständigen Operntext gehalten wird. Nichtsdestotrotz wird im Kapitel versucht, Eislers Gesamtvorhaben anhand der überlieferten Skizzen darzulegen. Daneben führt das Kapitel eine kontrastive Analyse von Doktor Faustus und Johann Faustus durch und geht auf stilistische und inhaltliche Kontinuitäten und Differenzen ein. $\mathrm{Zu}$ den wichtigsten Gemeinsamkeiten zählt die MontageTechnik, die durch verschiedene Bezüge auf die politische, gesellschaftliche und religiöse Geschichte Deutschlands sowie durch Stilmischungen zum Tragen kommt. Unter den Differenzen ist an erster Stelle die Einordnung der beiden Werke in die Bearbeitungen des Faust-Stoffes zu erwähnen: Dank der Präsenz der Figuren Faust und Mephisto sowie dem expliziten Charakter des Teufelspaktes lässt sich diese Einordnung bei Eisler anders als bei Thomas Mann kaum bestreiten.

Von der Intermedialität aus betrachtet, lässt sich festhalten, dass Eislers Johann Faustus nicht als intermediale Transposition, sondern eher als intermediale Bezugnahme auf Thomas Manns Roman zu werten ist, da Doktor Faustus lediglich zu den Vorlagen des Werkes zählt. Aus diesem Grund befinden sich die Ausführungen zu Johann Faustus noch im ersten Teil der vorliegenden Studie nach dem Kapitel zu den Forschungszielen und der Methode. Die Analyse führt in die Praxis des Medienvergleichs ein, die charakteristisch für diese Untersuchung ist. Zudem macht sie auf die Wichtigkeit der Berücksichtigung des Entstehungskontextes des sekundären intermedialen Produktes aufmerksam, was einen Leitgedanken der darauf folgenden Kapitel von Teil zwei und drei darstellt. 


\subsection{Hanns Eislers Johann Faustus und Thomas Manns Doktor Faustus: Kontinuitäten und Differenzen}

Vor der kontrastiven Analyse wird zunächst einmal die Debatte um Hanns Eislers Werk kurz umrissen, um die Entstehungsbedingungen des Werkes zu veranschaulichen. 1952 veröffentlicht der Aufbau-Verlag in Berlin das Libretto von Johann Faustus, welches jedoch aufgrund des raschen Druckverbots in der DDR nur kurze Zeit auf dem Markt bleibt. Das Verbot ist die Konsequenz langer Diskussionen, die vor allem an der Akademie der Künste in Berlin stattfinden. Laut Gerhard Müller hätte die Oper das „Magnum opus“ ${ }^{1}$ des Komponisten werden sollen. Das sind jedoch nur Vermutungen, denn nach den heftigen Kritiken verzichtet Eisler darauf, sich mit dem Werk weiter zu beschäftigen und die Musik zu komponieren: Ausnahme sind einige Skizzen und Fragmente, die man an der Berliner Akademie der Künste einsehen kann.

$\mathrm{Zu}$ den erbittertsten Kritiker*innen Eislers der damaligen Zeit zählen Wilhelm Girnus und Alexander Abusch. Der eine ist der Ansicht, die Geschichte des deutschen Volkes sei bei Eisler als eine der Reaktion und als eine ausschließlich negative dargestellt. ${ }^{2}$ Der andere findet im Operntext zahlreiche Widersprüche und kommt zur Aussage, dass Eisler mit dem Johann Faustus ,die geistige und dichterische Bedeutung von Goethes Werk für die deutsche Nationalliteratur und für die Geschichte des deutschen Volkes bagatellisiert ${ }^{* 3}$ habe. Auch die Verteidigung des Werkes etwa durch Lion Feuchtwanger, Bertolt Brecht und Leonhard Frank ändert nichts an der Situation. ${ }^{4}$ Dieses Kapitel beschäftigt sich also mit einem Werk, das wahrscheinlich noch kontroverser als Manns Doktor Faustus ist und so Hans Bunge - „totdiskutiert“5 wurde. Als Beleg für Bunges Aussage reicht

\footnotetext{
${ }^{1}$ Müller, Gerhard: Ein deutsches Trauerspiel. Die Debatte über Eislers Johann Faustus. In: Eisler-Mitteilungen 30 (2002), S. 9-11, hier: S. 9.

${ }^{2}$ Zit. in Eisler, Hanns: [Notizen zur Faustus-Polemik IV] (1953). In: MuP, S. 295 f., hier: S. $295 \mathrm{f}$.

${ }^{3}$ Zit. in Bunge, Hans: Die Debatte um Hanns Eislers „Johann Faustus“. Eine Dokumentation (Reihe Brecht-Studien). Hrsg. v. Brecht-Zentrum Berlin. Berlin: BasisDruck 1991, S. 56. Während seines zweijährigen Berufsverbots verfasste Bunge diese Dokumentation, auf die hier für eine vollständige Rekonstruktion der Debatte verwiesen wird. Der Vortrag von Abusch erschien auch in Sonntag (17.05.1953) und in Sinn und Form $3+4$ (1953) unter dem Titel „Faust - Held oder Renegat in der deutschen Nationalliteratur“.

${ }^{4}$ Vgl. Bunge: Die Debatte, S. 82; Eisler: [Stellungnahme zu den 6 Fragen von Wilhelm Girnus] (1953). In: MuP, S. 296-302, hier: S. 299.

${ }^{5}$ Bunge: Zu dieser Ausgabe. In: Eisler: Johann Faustus. Fassung letzter Hand. Hrsg. v. Hans Bunge, mit einem Nachwort v. Werner Mittenzwei. Berlin: Henschelverlag 1983,
} 
vielleicht bereits die Beobachtung, dass bis 1982 in der DDR keine Möglichkeit bestand, den Text aufzuführen. ${ }^{6}$

Thomas Manns Doktor Faustus ist eine der Vorlagen von Eislers Johann Faustus, die Hauptvorlage stellt jedoch das Puppenspiel dar. Im Rahmen der vorliegenden Arbeit wird das Libretto als eine der ersten Reaktionen auf Manns Roman betrachtet: Die Bezeichnung, Vertonung ' oder ,intermediale Transposition" scheint in diesem Fall nicht wirklich adäquat, weswegen die Definition ,intermediale Bezugnahme' präferiert wird. Auch für diesen Vergleich greift diese Studie auf das Forschungsparadigma der Intermedialität zurück: Laut der Definition von Medium, der sie folgt, ist ein Libretto Bestandteil des plurimedialen Mediums Oper. ${ }^{7}$ Zudem besteht in der Forschungsliteratur keinerlei Konsens was im Folgenden aufgezeigt werden soll - ob Eislers Werk für selbständig zu halten ist. Bereits diese erste Analyse zeigt, wie wichtig die Anwendung von Kernkategorien intermedialer Theorien auf konkrete Beispiele ist. In dieser Hinsicht versucht dieses Kapitel, Manns Roman und Eislers Libretto auf der Suche nach Kontinuitäten und Differenzen miteinander zu vergleichen. Dabei zieht es auch die Skizzen für die Musik in die Analyse ein: Auch deswegen plädiert das Kapitel für das Begriffsinventarium der Intermedialität, da das gesamte Opernprojekt in den Blick genommen wird. Diese Gesamtperspektive ermöglicht eine Einführung in den Prozess der Entstehung eines musikalischen Bühnenwerkes, das einen oder mehrere literarische Texte vertont. ${ }^{8}$ Als sekundäres Produkt regt Eislers Johann Faustus zudem zu Reflexionen über Aspekte an, die sich primär mit der Kontextualisierung des im Nachhinein entstandenen Werkes verbinden und die für die Analyse solcher Phänomene sehr ergiebig ist.

S. 5 ff., hier: S. 5. Diese Ausgabe des Librettos enthält die Korrekturen, die Eisler nach den Polemiken anfertigte.

${ }^{6} 1982$ wurde er im Berliner Ensemble unter der Regie von Manfred Wekwerth aufgeführt - früher dreimal in der BRD (1974 in Tübingen, 1976/77 in Kiel und WestBerlin) - und 1983 erschien das Libretto beim Henschelverlag (siehe Fußnote 5). Vgl. Schebera, Jürgen: Nachbemerkung. /Johann Faustus/ Oper ohne Musik/. In: Eisler: Johann Faustus, Leipzig: Faber \& Faber 1996, S. 147-166, hier: S. 166.

${ }^{7}$ Geht man aber von einer technisch-materiellen Definition von Medium aus, so wäre dies ein Beispiel für Intramedialität/Intertextualität. Vgl. 1.1.2.

${ }^{8}$ Zur Kritik an der Auffassung, ein Komponist vertone ein Libretto siehe Stollberg: Kombination von Literatur und Musik, S. 70. 


\subsubsection{Eislers Libretto: ein selbständiges Werk?}

Wie eingangs des Abschnitts angesprochen, gilt es zunächst einmal der Frage nachzugehen, ob man Eislers Text für selbständig halten kann. Die Problematik ist sehr umstritten und verknüpft sich auch mit der Gattung des Librettos selbst. ${ }^{9}$ Eisler schreibt dazu: ${ }^{10}$

Ich halte [...] die Hauptidee des Faustus für eine höchst aktuelle, für eine höchst kämpferische, für eine höchst fortschrittliche Idee, die sich natürlich in der Musik noch viel wuchtiger offenbaren muß als im Textbuch.

Brecht bestätigt diese Auffassung, laut der Eislers Projekt der Musik bedarf, in den Thesen zur Faustus-Diskussion, die er im Mai 1953 der MittwochGesellschaft der Akademie der Künste vorstellt. Brecht zeigt sich aber vom Libretto selbst sehr begeistert: ${ }^{11}$

Obgleich das Werk zu seiner vollen Wirkung der Musik bedarf, ist es ein bedeutendes literarisches Werk durch sein großes nationales Thema, durch die Verknüpfung der Faust-Figur mit dem Bauernkrieg, durch seine großartige Konzeption, durch seine Sprache, durch seinen Ideenreichtum.

Beide Zitate helfen zu erinnern - vergleichbar zur relativ einseitigen Definition eines Librettos als „,bloßen Sprungbrett[s] für musikalisch bzw. sängerisch hergestellte Affekterregungen“ _ ${ }^{12}$ dass, so Arne Stollberg, ,sich das Zusammenwirken von Musik und Text auf der Bühne im Moment der Live-Performance anders darstellt als auf dem Papier“. ${ }^{13}$ Die Forscher*innen, die Hanns Eislers Johann Faustus untersucht haben, vertreten hinsichtlich der Definition des Operntextes unterschiedliche Auffassungen. Hans Joachim Kreutzer erörtert: „Dieses Libretto [...] bedarf der Musik, als Drama, es ist kein bloßer ,Text ${ }^{\star}$, sondern es enthält

\footnotetext{
${ }^{9}$ Dazu siehe: Gier, Albert: Das Libretto. Theorie und Geschichte einer musikoliterarischen Gattung. Darmstadt: Wissenschaftliche Buchgesellschaft 1988, S. 3-32.

${ }^{10}$ Eisler: [Entwurf einer Stellungnahme zur Faustus-Polemik] (1953). In: MuP, S. 284-287, hier: S. 285.

${ }^{11}$ Zit. in Bunge: Die Debatte, S. 159.

${ }^{12}$ Stollberg: Kombination von Literatur und Musik, S. 71.

${ }^{13}$ Ebd.
} 
einen Freiraum, der auf Musik hin konzipiert ist“ “. ${ }^{14}$ Friederike Wißmann erläutert hingegen, Eisler habe „das Libretto als einen für sich stehenden Text ${ }^{\text {“15 }}$ verfasst, der ohne Musik nicht „defizitär" ${ }^{\text {“16 }}$ bleibe. Sie unterstreicht außerdem, dass die Absenz der Musik es jedoch nicht erlaube, den Text in der gleichen Weise wie ein Theaterstück zu behandeln: Das Libretto bediene sich stilistischer Register, „die seinem kompositorischen Verfahren erwachsen“. ${ }^{17}$ Dieser letzten Auffassung schließt sich auch die vorliegende Studie an: Dies mag auch die Tatsache bestätigen, dass der Operntext veröffentlicht wurde, bevor die Musik fertig war. Zwei Komponisten konnten ihn zudem vertonen: Karl Heinz Füssl (19661967), allerdings nur fragmentarisch, und Friedrich Schenker (2004). Der eine „wählt eine strenge dodekaphone Gestaltung“"18 und betont somit den Einfluss von Thomas Manns Roman, der andere komponiert ,dezidiert unter dem Aspekt der Textverständlichkeit ${ }^{\star 19}$ und stellt daher Eislers Text in den Vordergrund.

\subsubsection{Eislers Johann Faustus und Manns Doktor Faustus: Kontinuitäten}

Nach der Definition des Status des Operntextes wird im Folgenden auf die Kontinuitäten zwischen Eislers Werk und Manns Roman eingegangen. Mit diesem Zweck soll zunächst einmal die Beziehung zwischen Mann und Eisler knapp dargestellt werden. In den Exiljahren hatten die beiden Autoren die Möglichkeit,

\footnotetext{
${ }^{14}$ Kreutzer, Hans Joachim: Faust. Mythos und Musik, München: Beck 2003, S. 153.

${ }^{15}$ Wißmann, F.: Johann Faustus. Eislers Materialien und die Komposition des Textes. In: Schweinhardt, Peter (Hrsg.): Hanns Eislers „Johann Faustus“. 50 Jahre nach Erscheinen des Operntexts 1952. Symposion (Eisler-Studien Bd. 1). Wiesbaden: Breitkopf \& Härtel 2005, S. 11-25, hier: S. 11.

${ }^{16}$ Ebd., S. 12.

${ }^{17}$ Dies.: Faust im Musiktheater des 20. Jahrhunderts, S. 95. Immerhin besteht auch die Auffassung, eine Oper weise größere Affinitäten zum Roman als zum Drama auf. Siehe Stollberg: Kombination von Literatur und Musik, S. 70 und Conrad, Peter: Romantic Opera and Literary Form. Berkeley (u. a.): University of California Press 1977. Allerdings ist Theaterregisseur*innen auch gelungen, das Libretto aufzuführen, als wäre es als Theaterstück konzipiert worden. Siehe Fußnote 6.

${ }^{18}$ Wißmann, Robert: Kompositionen für den Faust. Musik zu Hanns Eislers Johann Faustus. In: Eisler Mitteilungen 30 (2002), S. 8, hier: S. 8.

${ }^{19}$ Ebd.
} 
einander kennenzulernen; ${ }^{20}$ ein Brief von Thomas Mann an Hanns Eisler drückt Neugierde für sein Johann Faustus-Projekt aus: ${ }^{21}$

Dankt für die Übersendung des Librettos zu einer volkstümlichen Faust-Oper. Nennt die Formung des Faust-Stoffes „,sehr neu, sehr kühn, sehr eigentümlich“, die auch der Musik wie dem Theater recht entgegenkommt. Glaubt, daß das Ganze besonders durch die Musik, an die er sich schwer gewöhnen könne, „hübsch provokant“ werde, besonders durch den derben deutschen Humor, der besonders in der Figur des Hanswurst zum Ausdruck kommen werde.

Eisler fasst die zentrale Idee von Johann Faustus folgendermaßen zusammen: ${ }^{22}$

Wer sich gegen das Volk, die Bewegung des Volkes, die Revolution stellt, sie verrät, gegen sie einen Bund mit den Herren schließt, wird vom Teufel geholt ohne Gnade und Erbarmen; er wird vernichtet.

Zusätzlich zu den gedanklichen Affinitäten zwischen Manns Werk und Eislers Oper, die sich am vorigen Zitat ablesen lassen, gibt Eislers Bezeichnung seines Textes als ,Doktor Faustus' einen ersten Hinweis für seine Nähe zu Thomas Manns Roman: Sie taucht nicht nur in den ersten Skizzen der Arbeit auf, sondern auch im Jahr 1953, also zur Zeit der Veröffentlichung des Librettos und der Debatte um seine Druckerlaubnis. ${ }^{23}$ Mit Bewunderung äußert sich Eisler über

\footnotetext{
${ }^{20}$ Sehr wahrscheinlich schätzten sich die beiden hoch. Der Autor von Doktor Faustus schreibt beispielsweise in der Entstehung des „Doktor Faustus “ über den Komponisten: „Dagegen traf man im Hause Schönberg Hans [sic] Eisler, an dessen sprühendem Gespräch ich immer das heiterste Gefallen fand“ (Ent: 82). Vgl. Schebera: Nachbemerkung, S. 147. Für eine Biographie von Eisler sei hier auf die folgenden Publikationen verwiesen: Wißmann, F.: Hanns Eisler. Komponist, Weltbürger, Revolutionär. Mit einem Vorwort v. Peter Hamm. München: Elke Heidenreich 2012. Siehe auch Albert, Claudia: Adorno und Eisler - Repräsentanten des Musiklebens in den beiden deutschen Staaten der Nachkriegszeit. In: Exilforschung - Ein internationales Jahrbuch Bd. 9 (1991), S. 68-80.

${ }^{21}$ Briefe 4, 05.11.1952, an Eisler (Mann diktierte seine Briefe seiner Sekretärin, daher die Verwendung der dritten Person Singular und der indirekten Rede). F. Wißmann, die aus demselben Brief im Archiv der Akademie der Künste zitiert, unterstreicht aber, dass oft nur „die Einschätzung ,hübsch provokant““ gelesen wird, nicht aber ,das Infragestellen [von Seiten Thomas Mann] des Librettotextes als einerseits ,unsingbar', andererseits ,wunderartig-merkwürdig““.Wißmann, F.: Eislers Johann Faustus: ,unsingbar“, ,unkomponierbar“, ,wunderbar-merkwürdig“. In: Faust-Jahrbuch 2 (2006), S. 23-34, hier: S. 25.

${ }^{22}$ Eisler: [Entwurf einer Stellungnahme], S. 284.

${ }^{23}$ Vgl. etwa Eislers Schriften „Notizen zu Dr. Faustus“ und „Vorbemerkung [zu Dr. Faustus]“ (1951, in: MuP, S. 136).
} 
Manns Roman und die Figur des Adrian Leverkühn, in der er vor allem seinen Lehrer Arnold Schönberg sieht: ${ }^{24}$

In großartiger Weise hat Thomas Mann verstanden, alle Krankheiten und (Probleme) Schwierigkeiten der modernen Musik und des (modernen) Musiklebens wie in einem Brennspiegel zu fangen und sie in seiner Gestalt des Leverkühn auf zwei Beine zu stellen.

Angeregt von der Lektüre von Doktor Faustus drückt Eisler in Skizzen und Notizen zu seinem Werk die Absicht aus, mit dem Johann Faustus eine Oper zu schreiben, die ,,von den unerfahrenen Ohren und den erfahrensten“ 25 begriffen werden müsse. Das Libretto setzt sich, wie bereits erwähnt, nicht nur mit Manns Roman auseinander, sondern geht - so Tim Lörke - ,,an die Anfänge des Faust-Mythos“"26 zurück.

Hier sei vor allem auf die inhaltlichen und strukturellen Parallelen zwischen Johann Faustus und Doktor Faustus hingewiesen. In Manns Roman kann man den Einfluss von Dantes Inferno mehrfach erkennen, etwa an der Schilderung der Komposition Apocalipsis cum figuris und am Zitat vor Beginn des Romans. Der Bezug auf die Commedia fehlt auch bei Eisler nicht, z. B. im Vorspiel durch die Einführung von Charon, dem ,treue[n] Totenfährmann“ (JF: 11). ${ }^{27}$

Auch das Motiv der Verzweiflung, die in Thomas Manns Roman als Voraussetzung für die Hoffnung gilt und Leverkühns Weheklag auf vielfältige Art und Weise darstellt, spielt in Johann Faustus eine wichtige Rolle. ${ }^{28}$ Noch im Vorspiel erklärt Mephisto, Faust sei ,,aus Verzweiflung viermal Doktor“ (JF: 17) geworden. Auch die seelische ,Verwirrung“ (JF: 123) Fausts, von der Wagner in der fünften Szene des dritten Aktes berichtet, ist nichts anderes als tiefe Verzweiflung, die - wie in Doktor Faustus - zu einem Geständnis führt. Während dieser confessio wiederholt Faust das Wort „Wehklagen“ (JF: 126 und 127). ${ }^{29}$ Damit wird auf

\footnotetext{
${ }^{24}$ Eisler: Notizen zu Dr. Faustus, S. 129 (Ergänzungen i. O.). ${ }^{25}$ Ebd., S. 133.

${ }^{26}$ Lörke, Tim: Die Verteidigung der Kultur. Mythos und Musik als Medien der Gegenmoderne. Thomas Mann - Ferruccio Busoni - Hans Pfitzner - Hanns Eisler. Würzburg: Königshausen \& Neumann 2010, S. 276.

${ }^{27}$ Siehe Alighieri, Dante: La commedia, Die göttliche Kömodie, I Inferno - Hölle. Übers. u. komm. v. Hartmut Köhler. Stuttgart: Reclam 2010, Canto III, V. 78-136, S. 50-55.

${ }^{28}$ Vgl. Kap. 5.

${ }^{29}$ Er wiederholt auch den Satz „da klagt doch wer“ (JF: 126 ff.) und benutzt einmal das grammatikalisch verwandte Wort „Anklagen“ (JF: 128). Seine confessio scheint auch vergleichbar zu Leverkühns Kantate - ein „Variationenwerk der Klage“ (DF: 705) zu sein.
} 
Leverkühns letztes Werk angespielt, in dem - so Zeitblom im Roman - „die letzte Verzweiflung Ausdruck“ (DF: 711) erhält und wo ebenfalls ein Geständnis folgt. Eisler sah für die Musik von Johann Faustus drei Lamentationes und eine Verzweiflungsarie im dritten Akt vor, was diese Bezugnahme noch verstärkt hätte. ${ }^{30}$ Sowohl bei Eisler als auch bei Thomas Mann scheint die letzte (oder die einzige?) Hoffnung in der Musik zu liegen. Das Lied des Knaben am Ende des Librettos, das im Folgenden zitiert wird, erinnert an das „hohe g eines Cello“ (DF: 711) am Ende von Leverkühns Weheklag:

Ich ging auf dürrer Heiden, / Da hört ein Stimm ich singen, / Tät mir wunderbar erklingen: / „Komm, lieber Tag; / Geh, finstre Nacht! / Fried und Freud / Und Freundlichkeit erwacht“. (JF: 144) ${ }^{31}$

Johann Faustus studiert wie Adrian Leverkühn als erste Disziplin die Theologie, die er dann aufgibt. ${ }^{32}$ Auch erleben beide eine Schaffenskrise, die sie zum Pakt mit dem Teufel führt:

MEPHISTO: Der Herr Doktor befindet sich in einer großen Schaffenskrise, fühlt sich abgestumpft gegen geistige wie körperliche Genüsse. Wissenschaften erscheinen ihm schal; Vernunft ekelt ihn. Er lechzt nach einem „vollen Leben“. (JF: 17)

Eislers Mephisto zeichnet sich genau wie Manns Teufel dadurch aus, dass er verschiedene Gestalten annehmen kann: Er erscheint beispielsweise ,in bürgerlicher Kleidung“ (JF: 43), „,in der Tracht eines Junkers“ (JF: 48) und als italienischer Sekretär. ${ }^{33} \mathrm{Zu}$ den Bedingungen des Paktes zählt im Libretto ebenfalls das Liebesverbot, das - wie bei Mann - eine wichtige Rolle spielt:

MEPHISTO: Zum dritten: Du mußt der Liebe entsagen!

FAUST: Der Liebe?

\footnotetext{
${ }^{30}$ Vgl. Eisler: [Musikalische Disposition für die Faustus-Oper] (1951). In: MuP, S. 136 f., hier: S. 137.

${ }^{31}$ Siehe dazu auch Giovannini, Elena: Il patto col diavolo nella letteratura tedesca dell'esilio. Rom: Aracne 2010, S. 321.

${ }^{32}$ Vgl. ebd., S. 294.

${ }^{33}$ Siehe dazu JF: 109 und Giovannini: Il patto col diavolo, S. 296 (Fußnote 84). Die Erscheinung des Teufels als italienischer Sekretär könnte außerdem auf das Teufelsgespräch in Palestrina in Manns Roman anspielen. Vgl. Kap. 6.
} 
MEPHISTO: Wichtigster Punkt im Vertrag! (JF: 51) $)^{34}$

Bei der Besiegelung des Vertrags zittert Faust, genau wie Leverkühn beim Teufelsgespräch (währenddessen er jedoch keinen Vertrag tatsächlich unterschreibt):

MEPHISTO: Hier der Vertrag. Erst dein Blut! Zieht mit seiner Hahnenfeder Blut aus Fausts Arm. Unterschreib!

FAUST: nimmt die Feder, seine Hand zittert.

MEPHISTO: Wie du zitterst, ich will deine Hand führen. Mephisto führt ihm die Hand und steckt den Vertrag in die Tasche. (JF: 56) ${ }^{35}$

Ein weiteres wichtiges Motiv, das sich sowohl in Doktor Faustus als auch in Johann Faustus findet, ist das des Deutschtums und der Auseinandersetzung mit der Geschichte Deutschlands unter verschiedenen Gesichtspunkten (religiösen, politischen, kulturellen): Ernst Fischer stellt 1952 fest, Faust sei bei Eisler „Zentralgestalt der deutschen Misere“"36 und sein Werk ,,die Tragödie eines Volkes“. 37 Der Komponist betrachtet diese nicht nur als eine historische, sondern auch als eine ,höchst aktuelle“ 38 weil er in der ,Spaltung Deutschlands“39 die „,nationale Misere“40 seiner Zeit sieht. In diesem Prozess der Auseinandersetzung mit der deutschen Geschichte darf Martin Luther, ,,[d]ie Leuchte der [...] Nation“ (JF: 125), nicht fehlen: Die Handlung spielt in seiner Epoche, d. h. die erzählte Zeit ist nicht die von Doktor Faustus. Zudem befindet sich Fausts Palast in Wittenberg und er bewundert in einigen Momenten seines Lebens Luthers Lehre, von dem er vor der confessio auch umarmt wird. Luther scheint für die Widersprüche

\footnotetext{
${ }^{34}$ Später erklärt Mephisto Faust, dass er nur sich selbst lieben darf. Vgl. JF: 53; zum Thema des Liebesverbots siehe auch: Giovannini: Il patto col diavolo, S. 282.

${ }^{35}$ Das Verhältnis Plutos zu Mephisto entspricht dem Mephistos zu Faust: Der Teufel ist Johann Faustus gegenüber besonders autoritär, während Johann Faustus fast im ganzen Libretto passiv bleibt. Siehe auch Giovannini: Il patto col diavolo, S. 297. Giovannini meint, beim Unterschreiben zittere Faust aufgrund einer psychologischen Spannung und nicht aus Kälte wie Leverkühn. Vgl. ebd., S. 285. Das Zittern ist ein wiederholtes Motiv von Doktor Faustus: Auch Zeitblom zittert oft beim Berichten über das Leben seines Freundes. Siehe z. B. DF: 324.

${ }^{36}$ Fischer, Ernst: Doktor Faustus und der deutsche Bauernkrieg. In: Sinn und Form 4 (1952) H. 6, S. 59-73, hier: S. 63, Herv. i. O.

${ }^{37}$ Ebd., S. 67, Herv. i. O.

${ }^{38}$ Eisler: [Entwurf einer Stellungnahme], S. 285.

${ }^{39}$ Ders.: [Stellungnahme zu den 6 Fragen], S. 297.

${ }^{40}$ Ebd.
} 
der deutschen Geschichte zu stehen, weil er sich gegen „,den päpstlichen Mißbrauch“ (JF: 127) äußert und die Bibel ins Deutsche übersetzt, damit sie auch von den Bauern verstanden werden kann. Die Bauer verrät er jedoch wie Faust, indem er die Herren verteidigt. Das Libretto denunziert also die Unmöglichkeit seines Volkes, eine Revolution zu Ende zu führen.

Sowohl Thomas Mann als auch Hanns Eisler bedienen sich der MontageTechnik. ${ }^{41}$ Der Komponist sieht sie in seinen Skizzen und Notizen zur Oper auf verschiedenen Ebenen vor. Zuerst auf der musikalischen: Zwar bezeichnen sowohl Fischer als auch Brecht den Operntext als eine Tragödie, ${ }^{42}$ indem sie vor allem auf die politischen Bezüge hinweisen und ausschließlich anhand des Librettos argumentieren. Aus den Plänen geht jedoch ein unterschiedliches Vorhaben hervor, was hauptsächlich die Musik hätte verdeutlichen sollen. Es handelt sich um die Mischung von drei Opernstilen, die entweder bestimmten Figuren oder gewissen Szenen zugeordnet werden: ${ }^{43}$

1. der Spieloper (Hanswurst - Gretel)

2. der seriösen Oper (Faust - Mephisto)

3. der komischen Oper (,Vorspiel in der Hölle und Hanswurstszenen“).

Eine Reflexion über soziale Klassen verknüpft sich folglich mit entsprechenden musikalischen Formen. Eisler schreibt dazu: „Die Gegenüberstellung des Volksliedes - gegen den Kirchenchoral“. Die Macht als potestas, also unter ihren negativen und restriktiven Aspekten gefasst, ${ }^{44}$ die sich auch durch das Vorschreiben bestimmter musikalischer Formen in gewissen Kontexten ausdrückt, steht im Zentrum von Eislers Vorhaben. Durch die Mischung von Stilen und Formen in der Musik des Orchesters - von einigen Forscher*innen als Erzählinstanz der Oper angesehen ${ }^{45}$ - könnte es aber auch mit einer Art variabler und manchmal multipler Fokalisierung mit unterschiedlichen Erzählinstanzen in Verbindung gebracht

\footnotetext{
${ }^{41} \mathrm{Zu}$ Eislers Auseinandersetzung mit dem Erbe und den geteilten Meinungen zwischen ihm und Lukács, was das Montage-Verfahren angeht, vgl. Wißmann, F.: Johann Faustus, S. $16 \mathrm{f}$.

${ }^{42}$ Vgl. Fischer: Doktor Faustus, S. 67 und Bunge: Die Debatte, S. 159.

${ }^{43}$ Eisler: [Zur Wirkung der Faustus-Oper auf die Musikentwicklung] (1951). In: MuP, S. 137 f., hier: S. 137.

${ }^{44}$ Dazu siehe Braidotti, Rosi: Intensive Genre and the Demise of Gender. In: Angelaki. Journal of the Theoretical Humanities 13 (2008) H. 2, S. 45-57, hier: S. 56 Note 1.

${ }^{45}$ Vgl. Stollberg: Kombination von Literatur und Musik, S. 70.
} 
werden, die nicht ausschließlich die damaligen Macht legitimierenden Akteure repräsentieren. ${ }^{46}$

Eine weitere Ebene des Montage-Verfahrens ist die sprachliche. Zum einen orientiert sich Eisler wie Mann an einer Vielzahl von Vorlagen, z. B. an den Puppenspielen, dem Volksbuch, sowie an Goethe und Mann, um nur einige Quellen zu benennen. ${ }^{47}$ Zum anderen erinnert der Sprachgestus ,,an Hans Sachs und d[ie] Luthersche[] Bibelübersetzung “48 und an Autor*innen des 20. Jahrhunderts, nicht nur an Mann, sondern auch an Brecht. ${ }^{49}$ „Eisler verbindet in seinem Faustus-Text die Zeit des Bauernkrieges mit Erlebnissen aus dem Exil des 20sten Jahrhunderts, ohne eine sprachliche Vereinheitlichung zu bemühen“",50 so F. Wißmann, die dadurch auf eine weitere Ebene der Montage-Technik hinweist, welche die historische Zeit betrifft. Die Handlung spielt zwar zur Zeit der Bauernkriege, der Text jedoch ist reich an Anspielungen auf den Nationalsozialismus, den Holocaust, die deutsche Schuld, die Diskriminierung der Schwarzen in den USA, den Kalten Krieg und die DDR. In Doktor Faustus gibt es kraft der Extra- und Intradiegese zwei erzählte Zeiten, d. h. die Zeitbloms und des Zweiten Weltkriegs und die Leverkühns vor 1940; anhand von Digressionen und Andeutungen wird aber über weitere Ereignisse der abendländischen Geschichte reflektiert.

\footnotetext{
${ }^{46}$ Siehe Jahn, Manfred: Focalization. In: Herman, David, Jahn, Manfred u. Marie-Laure Ryan (Hrsg.): Routledge encyclopedia of narrative theory. London/New York: Routledge 2005, S. 173-177; Genette, Gérard: Die Erzählung. München: Fink 1994, S. 134-138; Foucault, Michel: Subjekt und Macht (1982). Übers. v. Michael Bischoff. In: Ders.: Schriften in vier Bänden. Dits et Ecrits. Bd. IV 1980-1988. Hrsg. v. Daniel Defert und François Ewald unter Mitarbeit v. Jacques Lagrange. Frankfurt am Main: Suhrkamp 2005 [Chicago 1982], S. 269-294.

${ }^{47}$ Vgl. dazu: Wißmann, F.: Johann Faustus, S. 13. Darüber hinaus ist der Einfluss Brechts sehr deutlich, was etwa an der Verwendung des Verfremdungseffekts zu erkennen ist. Nachdem beispielsweise Mephisto Fausts Seele genommen hat, wendet er sich zum Publikum und sagt: „Aplaudite, amici! Contractum finitum est“, JF: 142. Zum Thema siehe auch Giovannini: Il patto col diavolo, S. 320; und Wißmann, F.: Johann Faustus, S. 17. ${ }^{48}$ Wißmann, F.: Johann Faustus, S. 14.

${ }^{49}$ Siehe beispielsweise JF: 28: „Dann gewürzte Leberwurst, / Bratwürst auch und Schwartenmagen, / Erbsenbrei und Schweinebraten, / Braunbier nicht vergessen! / Das wär Essen!“. Die Worte erinnern an Brecht/Weills Oper Aufstieg und Fall der Stadt Mahagonny. Siehe Weill, Kurt und Bertolt Brecht: Aufstieg und Fall der Stadt Mahagonny. Oper in drei Akten. Leipzig: Universal-Edition 1929. Darüber hinaus spielen sowohl das Libretto Brechts als auch das Eislers in einer amerikanischen Stadt, obwohl sie im ersten Fall fiktiv ist.

${ }^{50}$ Wißmann, F.: „unsingbar“, S. 32.
} 
Auch bei Eisler könnte man von zwei Hauptfiguren sprechen: Faust und Hanswurst. ${ }^{51}$ Diese unterscheiden sich nicht wie bei Mann dadurch, dass sie zu zwei unterschiedlichen Konfessionen gehören oder unterschiedliche Auffassungen über die Kunst vertreten. Vielmehr trennen sie die soziale Klasse und die Ausbildung: Faust, Sohn eines Bauern, hat viel studiert und besitzt einen Palast in Wittenberg, Hanswurst ist ein armer, wenig gebildeter „Fresser“. 52

Wißmann erläutert diesbezüglich: ${ }^{53}$

Zum einen kann er [Eisler] durch Hanswurst den Text im Text kommentieren, andererseits behält die Figur auch ihre Aufgabe bei, politische Mißstände in ironischen Seitenhieben sichtbar zu machen.

Hanswurst teilt deshalb mit Zeitblom die kommentierende Funktion der Geschehnisse. Fausts breiteres kulturelles Vermögen ermöglicht ihm aber nicht, vernünftiger als Hanswurst zu handeln: Beide gehen einen Pakt mit dem Teufel ein und sind verdammt, weil sie nur an sich selbst gedacht haben. Ihre fehlende altruistische Natur macht sie ähnlicher und komplementärer als erwartet und nivelliert die Klassenunterschiede: Laut Giovannini verkörpern sie beide Seiten eines negativen Ichs $;{ }^{54}$ auch Brecht spricht von einem ,dunklen Zwilling“"55 von Goethes Faust. Mehr als in Doktor Faustus stehen aber Faust und Hanswurst in Johann Faustus „in einem fast komischen Gegensatz“" ${ }^{56}$ so Thomas Mann bezüglich Leverkühn und Zeitblom. Dieser „komische[] Gegensatz“57 lässt sich an den Namen der Figuren sofort ablesen. Zudem verdeutlichen die Namen, was Eisler laut seinen Skizzen auch in der Musik zu realisieren beabsichtigte, insofern sie sich sofort auf präzise literarische Traditionen, die Tragödie bzw. das Trauerspiel einerseits, die (Stegreif-)Komödie andererseits sowie auf präzise Autoren wie Goethe zurückführen lassen. Das Verhältnis dieser literarischen Traditionen zum Medium Musik, nämlich durch die Oper sowie die wechselseitigen Beziehungen zwischen Theater und Musiktheater, hätte Johann Faustus in Opernform wahrscheinlich noch deutlicher herausgestellt.

\footnotetext{
${ }^{51}$ Thomas Mann spricht in der Entstehung des „Doktor Faustus“ von den „,beiden Protagonisten“ (Ent: 72) von Doktor Faustus.

${ }^{52}$ Bunge: Die Debatte, S. 142.

${ }^{53}$ Wißmann, F.: Faust im Musiktheater, S. 93.

${ }^{54}$ Vgl. Giovannini: Il patto col diavolo, S. 313.

${ }^{55}$ Zit. in Bunge: Die Debatte, S. 161.

${ }^{56}$ GkFA 19.1: S. 261.

${ }^{57}$ Ebd.
} 
Aus den vorigen Ausführungen kristallisieren sich einige Gemeinsamkeiten zwischen Eislers und Manns Faustus-Projekt heraus, die sich auf verschiedenen analytischen Ebenen feststellen lassen. Erstens an den literarischen, politischen, religiösen und historischen Bezügen, z. B. auf Dante und Luther. Zweitens auf der Ebene der Figuren, etwa der drei Gestalten des Teufels oder der zwei Hauptfiguren der Werke. Drittens von einem stilistischen Gesichtspunkt aus, was hauptsächlich an der Montage-Technik erkennbar wird. Schließlich auf der Ebene der Motive, beispielsweise der Verzweiflung und des Teufelspaktes. Dieses letzte Motiv, zentrales Handlungselement einer Bearbeitung des Faust-Stoffes, leitet die kontrastive Analyse zum zweiten Schritt über, mit dem sich der nächste Unterabschnitt befasst, weil diesbezüglich signifikante Differenzen zwischen Manns und Eislers Text deutlich werden.

\subsubsection{Eislers Johann Faustus und Manns Doktor Faustus: Differenzen}

Wie am Ende des vorigen Unterabschnitts bereits angedeutet, treten beim Vergleich beider Texte in puncto Teufelspakt nicht nur Gemeinsamkeiten, sondern auch wichtige Differenzen zutage. Das fällt bereits bei der ersten Lektüre von Eislers Text auf: Das Libretto lässt die Leser*innenschaft nicht an der Existenz eines Teufels und eines Paktes (anders als bei Mann) zweifeln. Mephisto ist bei Eisler eine der Hauptfiguren des Werkes, die keine vage Identität wie in Doktor Faustus hat, welche die Konsequenz eines Deliriums sein könnte. Faust heißt nicht etwa Adrian Leverkühn, sondern eben Faust, und unterschreibt den Vertrag bewusst mit seinem Blut: Aus diesem Grund weist Giovannini darauf hin, dass in Johann Faustus der Pakt einen expliziten Charakter hat. ${ }^{58}$ Diese Beobachtungen zeigen, dass die Zuordnung von Eislers Werk innerhalb der Faust-Tradition nur schwer in Frage gestellt werden kann.

Die Dauer des Paktes beträgt in beiden Werken 24 Jahre. Der Unterschied ist aber, dass die Laufzeit in Doktor Faustus respektiert wird, in Johann Faustus dagegen nicht: Trotz des intensiven Studiums bleibt Faust für Mephisto der Sohn eines Bauern. Der Teufel folgt der calvinistischen Prädestinationslehre. ${ }^{59}$

\footnotetext{
${ }^{58}$ Siehe ebd., S. 279. Vgl. Kap. 6.

${ }^{59}$ Susanne Drees erläutert, das lateinische Wort praedestinatio bedeute im engeren Sinne „die Auffassung, dass das Schicksal eines Menschen von Gott vorherbestimmt ist“. Laut Calvin sei die Prädestinationslehre in der Heiligen Schrift begründet: Dieser Lehre entsprechend seien alle Menschen ,zum Heil oder Unheil“ prädestiniert. Folglich kann Johann Faustus weder seinen sozialen Status durch das Studium ändern, noch dem Teufel, der
} 
Johann Faustus verlangt vom Teufel, die Künste zu lernen:

FAUST: Zum vierten: Du sollst mich die Künste lehren.

MEPHISTO: Nenn sie!

FAUST: Die große Malerei, die hohe Musik, die edle Dichtkunst.

MEPHISTO: Das kann ich nicht.

FAUST: Du hast keine Macht über sie?

MEPHISTO: schweigt.

FAUST: Ich brauch den Ruhm. Die Herrn der Welt müssen vor mir sich neigen, die Sorg im Blick, ob ich auch guter Laun. Schlägt auf den Tisch. Wichtigster Punkt im Vertrag!

MEPHISTO: Durch eine neue Kunst, die ich dir lehr, kannst du Helden erscheinen lassen, als ob sie wirklich wären und lebeten. (JF: 49)

Hinsichtlich der Künste, die Johann Faustus vom Teufel gelehrt werden möchte, bestehen signifikante Unterschiede zwischen Eislers Libretto und Manns Roman. Eislers Faust will nicht nur die Musik lernen, sondern auch die Malerei und die Dichtkunst. Der Teufel aber ist kein „Musikintelligenzler“ (DF: 327) wie bei Mann und kann ihm nur „ein steriles Surrogat von Kunst bieten“60 - denn die Schwarzspiele sind lediglich „Illusionen von Kunst“. 61 Tim Lörke erläutert dazu: ${ }^{62}$

[A]lles, was Mephisto bieten kann, ist flüchtiges Blendwerk, weil es nicht der sozialen Lage angemessen ist. Ist Wahrheit einzig auf seiten des Volks zu finden, kann solche Kultur nur Lüge sein.

Eislers Teufel wirkt, wahrscheinlich weil er eher die Interessen der Herren als die des Volkes verteidigt, viel schwächer als der Teufel Thomas Manns. Nicht lediglich, weil er Faust keine echte Kunst bieten kann, sondern auch, weil er Plutos

ebenfalls einem von Gott gewollten Plan folgt, Bedingungen diktieren. Drees, Susanne: Prädestination und Bekenntnis. Die Rezeption der Prädestinationslehre Johannes Calvins in den europäischen reformierten Bekenntnisschriften bis 1619. Kamen: Spenner 2011, S. 22 u. 25.

${ }^{60}$ Lörke: Die Verteidigung der Kultur, S. 279.

${ }^{61}$ Ebd.

${ }^{62}$ Ebd. 
Untergebener ist. ${ }^{63}$ Die Hölle von Johann Faustus weist eine präzise Hierarchie auf, wovon man gleich im Vorspiel erfährt, wenn Pluto Mephisto befiehlt, ihm die Seele Fausts zu bringen. Pluto wird dort als autoritäre Figur dargestellt und Mephisto betont an mehreren Textstellen die Legitimationsinstanz seiner Macht. ${ }^{64}$ Zwar benutzt auch der „Teufel“ von Doktor Faustus mehrfach die erste Person Plural und weist so auf die Struktur der Hölle hin. ${ }^{65}$ Bei genauerer Betrachtung aber gewinnt man den Eindruck, dass er dort niemandem untergeben ist: Aus dieser Perspektive wäre die Verwendung der ersten Person Plural als pluralis majestatis der Herrscher*innen zu werten.

Ein weiteres Motiv, das in beiden Texten auftaucht, ist das Motiv der Krankheit, bei dessen Verwendung sich allerdings vor allem Unterschiede feststellen lassen. In Doktor Faustus soll - laut Zeitbloms Wiedergabe der Ereignisse - der Geschlechtsverkehr mit Esmeralda, durch den die Syphilis übertragen wird, mit der Unterschrift des Paktes korrespondieren. Die Symptome der Krankheit intensivieren sich während der Laufzeit des Vertrags bis hin zum Wahnsinn und zur Paralyse. Die Infektion stellt für den Erzähler den Impuls für viele Kompositionen Leverkühns dar. Ganz im Gegensatz zum Teufel in Manns Roman akzeptiert Eislers Mephisto die Bedingung Fausts, 24 Jahre gesund zu bleiben:

FAUST: Denn ich muß die vierundzwanzig Jahr in voller Gesundheit dahin leben.

MEPHISTO: Wenn du es reichlich bunt treibst mit Fressen, Saufen und Huren wie kannst du gesund bleiben? Aber da hab ich schon meine Hausmittelchen. Denkt eine Weile nach. (JF: 50)

Erst gegen Ende des Paktes zeigt Faust die Symptome einer psychischen Krankheit:

ARZT: Wie äußert sich die seltsame Verwirrung?

WAGNER: Seit sieben Tagen findet er keinen Schlaf, ächzend schleppt er sich durchs Haus. „Verhängt die Spiegel“, schreit er, „kann mein Gesicht nicht sehn.“ Dann wieder hockt er in einer Ecke und murmelt Flüche.

\footnotetext{
${ }^{63}$ Vgl. auch Giovannini: Il patto col diavolo, S. 281.

${ }^{64}$ Siehe etwa JF: 44: „Von Pluto habe ich die Macht, in jeglicher Gestalt zu erscheinen“, und JF: 108: „Sei ohne Sorg, durch meine Macht, von Pluto mir verliehn, mache ich aus deinem Wagner einen Mann, voll Phantasie, voll Schwung und Wärme“.

${ }^{65}$ Vgl. z. B. DF: 335: „Überall, wo das Stundglas gestellt und Zeit gegeben ist, unausdenkbare, aber befristetete Zeit und ein gesetztes Ende, da sind wir wohl auf dem Plan, da blüht unser Weizen“ (Herv. A. O.).
} 


\section{ARZT: Wem flucht er?}

WAGNER: Sich. (JF: 123) ${ }^{66}$

Diese „seltsame Verwirrung“ sieht nicht wirklich wie eine Krankheit, sondern eher wie tiefe Verzweiflung und Angst aus: Faust wird sich der Tatsache bewusst, dass er durch die Hilfe des Teufels nichts Besseres realisieren konnte. Zugleich begreift er, dass Mephisto ohnehin seine Seele haben würde: Der Pakt ist ungerecht und unausgewogen, der Teufel hat ihn verspottet. In Doktor Faustus gelingt es Adrian Leverkühn durch die Hilfe des Teufels ein neues kompositorisches System zu entwickeln, was manche jedoch ebenfalls für „Illusionen von Kunst“67 halten könnten. Gleichwohl könnte darauf hingewiesen werden, dass Adrian Leverkühn durch seine letzte Komposition eine größere Ausdruckskraft der Musik zu erreichen beabsichtigt, was aber die „Strenge“ der Form, d. h. die präzise Anwendung der Dodekaphonie, verhindert: In der Aufführungssituation wird das besonders deutlich, da der Künstler nach dem ersten Laut in Ohnmacht sinkt. ${ }^{68}$

Zweifelsohne besteht ein weiterer, signifikanter Unterschied zwischen Eislers Johann Faustus und Thomas Manns Doktor Faustus darin, dass im Libretto die Musik keinen wichtigen Bestandteil der histoire ${ }^{69}$ mehr darstellt: Die Oper rückt nicht das Leben eines Musikers ins Zentrum, sondern die Musik ist einfach eine der Künste, die Faust vom Teufel lernen will. Die histoire lässt sich bei Eisler eindeutig als eine Bearbeitung des Faust-Stoffes erkennen, und zwar nicht nur im paratextuellen Hinweis des Titels. ${ }^{70}$

Darüber hinaus ist eine Berücksichtigung des Entstehungskontextes, wie eingangs des Kapitelabschnitts angesprochen, für die Analyse sehr ergiebig: Das sekundäre Produkt wird nicht 1947 gleich nach Ende des Zweiten Weltkriegs in der BDR bzw. in Schweden, sondern 1952 in der DDR veröffentlicht. Während Thomas Manns Roman ein höchst kontroverses Werk bleibt, das aber weiterhin gelesen und veröffentlicht werden durfte bzw. darf, zu dem sich der Autor auch wiederholt äußert und sogar ein zweites Werk über seine Entstehungsgeschichte erscheinen lässt, erfährt Eislers Libretto eine andere Geschichte. Dieses wird 30 Jahre lang nicht veröffentlicht und die Musik dementsprechend nur sehr

\footnotetext{
${ }^{66}$ Siehe dazu auch Giovannini: Il patto col diavolo, S. 319.

${ }^{67}$ Lörke: Die Verteidigung der Kultur, S. 279.

${ }^{68}$ Vgl. DF: $728 \mathrm{f}$.

${ }^{69}$ Hier und im weiteren Verlauf der Studie aus: Genette, Gérard: Palimpsestes. La littérature au second degré. Paris: Seuil 1982, z. B. S. 319.

${ }^{70}$ Dazu vgl. auch Genette, G.: Paratexte. Das Buch vom Beiwerk des Buches. Frankfurt am Main: Suhrkamp 2019 [Paris 1987], S. 86.
} 
fragmentarisch komponiert. Die kritischen Reaktionen auf Eislers Werk, nach denen die Darstellung der deutschen Geschichte in Johann Faustus von Pessimismus und Einseitigkeit geprägt sowie Goethes Faust bagatellisiert sei, hatten Konsequenzen für die Verbreitung und die Vervollständigung des Werkes. ${ }^{71}$ Müller kommt sogar zur Aussage, dass Eislers Johann Faustus ,ein Beispiel für den kulturpolitischen Dogmatismus der DDR“72 sei. In der Rezeptionsgeschichte von Thomas Manns Doktor Faustus und Hanns Eislers Johann Faustus profilieren sich demnach wahrscheinlich die größten Unterschiede. Differenzen, die mit dem unterschiedlichen Entstehungsdatum und Veröffentlichungsort zusammenhängen, sind allerdings nicht nur werkextern, sondern auch werkintern an den politischen und historischen Anspielungen und Bezügen beider Texte zu erkennen. Auch diese sind historisch und geographisch bedingt und machen folglich eine (auch grobe) Kontextualisierung für die Analyse erforderlich.

\section{$2.2 \quad$ Fazit}

Das Kapitel hat anhand der Werke Doktor Faustus von Thomas Mann und Johann Faustus von Hanns Eislers gezeigt, unter welchen Aspekten die Analyse von Kontinuitäten und Differenzen zwischen Vorlage und intermedialer Bezugnahme durchgeführt werden kann. Im Gesamtkontext der Arbeit machte sie folglich auf einige wichtige Kriterien für den Medienvergleich aufmerksam, so z. B. den Entstehungskontext, stilistische Entscheidungen sowie das Verhältnis zum Faust-Stoff und zu den im Roman auftauchenden Motiven. Im Hinblick auf die Forschungsfragen der Studie ermöglichte die Analyse von Eislers Johann Faustus bereits ein Stück der frühen kompositorischen Rezeptionsgeschichte von Doktor Faustus zu rekonstruieren.

\footnotetext{
${ }^{71}$ Vgl. 2.1. Eine ausführliche Rekonstruktion der damaligen Debatte um Eislers Werk sowie aller Reaktionen auf Manns Roman würde den Rahmen dieser Untersuchung sprengen und auch zu weit vom intermedial angelegten Schwerpunkt abweichen. Daher wird diese Problematik im Rahmen dieser kontrastiven Analyse nur kurz umrissen. Für weitere Informationen zum Hintergrund sei jedoch auf die Publikationen von Bunge, F. Wißmann, Müller, Lörke und Giovannini, die in diesem Kapitel bereits zitiert wurden.

${ }^{72}$ Müller: Ein deutsches Trauerspiel, S. 9.
} 
Open Access Dieses Kapitel wird unter der Creative Commons Namensnennung - Nicht kommerziell - Keine Bearbeitung 4.0 International Lizenz (http://creativecommons.org/lic enses/by-nc-nd/4.0/deed.de) veröffentlicht, welche die nicht-kommerzielle Nutzung, Vervielfältigung, Verbreitung und Wiedergabe in jeglichem Medium und Format erlaubt, sofern Sie den/die ursprünglichen Autor(en) und die Quelle ordnungsgemäß nennen, einen Link zur Creative Commons Lizenz beifügen und angeben, ob Änderungen vorgenommen wurden. Die Lizenz gibt Ihnen nicht das Recht, bearbeitete oder sonst wie umgestaltete Fassungen dieses Werkes zu verbreiten oder öffentlich wiederzugeben.

Die in diesem Kapitel enthaltenen Bilder und sonstiges Drittmaterial unterliegen ebenfalls der genannten Creative Commons Lizenz, sofern sich aus der Abbildungslegende nichts anderes ergibt. Sofern das betreffende Material nicht unter der genannten Creative Commons Lizenz steht und die betreffende Handlung nicht nach gesetzlichen Vorschriften erlaubt ist, ist auch für die oben aufgeführten nicht-kommerziellen Weiterverwendungen des Materials die Einwilligung des jeweiligen Rechteinhabers einzuholen.

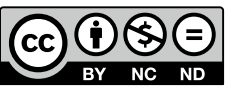

